\title{
Liquid Droplet Evaporation in Simulations of the Flow in Pulsed-Pressure MOCVD
}

\author{
C. -W. Lim ${ }^{\mathrm{a}}$, H. M. Cave ${ }^{\mathrm{a}, \mathrm{b}}$, M. C. Jermy ${ }^{\mathrm{a}}$ and S. P. Krumdieck ${ }^{\mathrm{a}}$ \\ ${ }^{a}$ Department of Mechanical Engineering, University of Canterbury, Private Bag 4800, \\ Christchurch 8140, New Zealand. \\ ${ }^{\mathrm{b}}$ Department of Mechanical Engineering, National Chiao-Tung University, Hsinchu, \\ Taiwan.
}

In this paper, a numerical model of the liquid precursor droplet flash evaporation and transport is introduced. This droplet model is coupled with the Quiet Direct Simulation (QDS) scheme to model the unsteady flow development in a Pulsed Pressure MOCVD reactor. A preliminary simulation of the PP-MOCVD process including the injection and pump down phase is conducted. The simulation results are consistent with the measured pressure change in the PP-MOCVD process.

\section{Introduction}

Pulsed-Pressure CVD (PP-CVD) is a new deposition technique which has demonstrated improved performance over traditional CVD technologies. PP-CVD reactor with liquid, rather than gas, precursor delivery systems are attractive as many precursor chemicals are available in soluble forms, particularly metalorganics. In fact, liquid-injection delivery systems have been used in the majority of PP-CVD deposition experiments. Xie and Raj (1) deposited lithium tantalite $(\mathrm{LiTaO} 3)$ on sapphire from a liquid metalorganic precursor of lithium hexa-t-butoxide. Versteeg et al. (2) then deposited titanium dioxide on sapphire from titanium tetra isoproxide (TTIP). Later, Krumdieck et al. (3) performed a deposition of yttria-stabilised zirconia (YSZ) films onto solid oxide fuel cell electrodes to act as an electrolyte layer.

The Pulsed-Pressure MOCVD (PP-MOCVD) process under development in our group uses timed injection of a controlled volume of liquid metalorganic precursor via an ultrasonic atomizer. The precursor is injected into a continuously evacuated reactor. The pressure varies rapidly with time and the precursor flow in the reactor chamber is unsteady. Experimental evidence has shown the resultant flow regime produces a uniform reactant mass flux within the reactor, which results in highly uniform thin films with high precursor conversion efficiencies $(4,5,6)$.

In the PP-MOCVD process, the injected liquid precursor droplets evaporate rapidly by flash vaporization. The vapour concentration is high near the reactor inlet during the injection phase. The continuously evacuated reactor chamber causes the fluid density to reduce significantly with time after the end of the injection phase, and with the distance from the inlet. Previous attempts to model PP-CVD with DSMC solvers have proved extremely challenging and computationally expensive (7) as well as being limited to pure gas phase simulation, i.e. unable to simulate the injection and evaporation of a liquid precursor solvent due to the very large density difference between the flow adjacent to the droplet and far from them. 
In order to continue to develop the liquid injection delivery system for PP-MOCVD, a greater understanding of the flow dynamics of the rapid evaporated liquid precursor and solvent in the unsteady pulsed pressure regime is essential. In this paper, a numerical model of the liquid precursor droplet evaporation and transport is presented. This liquid droplet model uses a Lagrangian tracking method to simulate a representative number of simulated droplets, from which the behaviour of the spray as a whole is extrapolated. Each liquid precursor droplet is injected into the computational domain with an initial droplet size and velocity selected stochastically from the log-normal distribution typical of sprays. Conservation of mass, momentum and energy equations combined with Maxwell's diffusion law (8) are solved for each simulated droplet.

In the simulation of the unsteady flow regime in a PP-MOCVD reactor, the liquid droplet model is coupled to a novel kinetic-based gas flow solver that utilizes the Quiet Direct Simulation (QDS) technique which has been demonstrated to be viable method for PP-MOCVD flow simulation $(9,10)$. Droplet models have been developed before for Navier-Stokes CFD solvers but this work reports the first implementation of a droplet model in a QDS flow solver. The liquid droplet model computes the vaporized mass, momentum and energy from the liquid droplets. These become source terms in the QDS solver, which simulates the gas dynamics in the PP-MOCVD reactor in turn. In the absence of more detailed experimental data on droplet evaporation phenomena, particularly at low pressures, the simulation results are compared to the predicted pressure change in the reactor and the deposited film uniformity shown in the experimental studies $(4,5)$.

\section{Method}

The flash evaporation model presented here models the evolution of the basic macroscopic properties (mass, momentum and energy) of a liquid droplet exposed to a low pressure vapour. The conservation equations of these properties are derived as the droplet model equations. The simulated droplet is assumed to be spherical with uniform temperature.

\section{$\underline{\text { Conservation of Mass }}$}

The mass rate of change of a simulated droplet is described with the equation of Pruppacher and Klett (11) with some modification for the present simulation conditions:

$$
\frac{d m}{d t}=\frac{4 \pi r D_{v}^{*} M}{R}\left(\frac{P_{g}}{T_{g}}-\frac{\Delta P}{T}\right)
$$

where $m$ is the mass of the droplet, $r$ is the droplet radius, $M$ is the molar mass, $R$ is the universal gas constant, $P_{g}$ and $T_{g}$ are the pressure and temperature of the surrounding gas respectively, $T$ is the droplet temperature, $\Delta P=2 \gamma / r$ is the Young-Laplace equation for pressure inside an equilibrium spherical droplet with a surface tension of $\gamma$, and $D_{v}{ }^{*}$ is the effective gas-phase diffusion coefficient given by Pruppacher and Klett (11) as; 


$$
D_{v}^{*}=\frac{D_{v}}{\frac{r}{r+\lambda}+\frac{4 D_{v}}{r \alpha \bar{v}}}
$$

where $\lambda$ is taken to be the mean free path of the surrounding gas, $\alpha$ is the mass accommodation coefficient, $\bar{v}$ is the mean molecular speed, and $D_{v}$ is the diffusion coefficient based on Enskog's theory of dense hard sphere fluids reported by Dariva et al. (12) which can be determined by the following equations:

$$
D_{v}=\frac{3 \bar{v}}{8 n \sigma^{2} g(\sigma)}
$$

where $n$ is the number density of surrounding gas, $\sigma$ is the hard-sphere diameter, and $g(\sigma)$ is the pair correlation function at the contact point for hard-spheres which is reported by Carnahan and Starling (13) as:

$$
g(\sigma)=\left|\frac{1-\frac{\xi_{3}}{2}}{\left(1-\xi_{3}\right)^{3}}\right| ; \xi_{3}=\frac{\pi n \sigma^{3}}{6}
$$

\section{Conservation of Momentum}

The momentum rate of change of a simulated droplet is calculated from the momentum exchange between the simulated spherical droplet and the surrounding gas, utilizing the drag coefficient. The change in droplet momentum, $p$, in a time step is:

$$
\frac{\partial p}{\partial t}=F_{\text {gas-drop }}=\frac{\vec{V}_{g}-\vec{V}}{\left|\vec{V}_{g}-\vec{V}\right|} C_{D} \frac{\pi d^{2}}{4} \frac{1}{2} \rho_{g}\left(\vec{V}_{g}-\vec{V}\right)^{2}
$$

where $F_{\text {gas-drop }}$ is the drag force exerted by the surrounding gas molecules to the droplet, $\rho_{g}$ and $\vec{V}_{g}$ are the density and velocities of the surrounding gas, respectively, $d$ is the droplet diameter, $\vec{V}$ is the droplet velocities, and $C_{D}$ is the drag coefficient given by Clift et al. (14) as:

$$
C_{D}=\frac{24}{\operatorname{Re}}\left[1+0.15 \operatorname{Re}^{0.687}\right]+\frac{0.42}{1+4.25 \times 10^{4} \mathrm{Re}^{-1.16}}
$$

which is valid for Reynold's number, $\mathrm{Re}<3 \times 10^{5}$ and spherical droplet with Weber number, $W e$, less than 2 . The Reynold's number can be determined by:

$$
\operatorname{Re}=\frac{\rho_{g} d\left|\vec{V}_{g}-\vec{V}\right|}{\mu_{g}}
$$

where $\mu_{g}$ is the viscosity of the surrounding gas. 


\section{Conservation of Energy}

The conservation of energy of a simulated droplet considers the net change of energy within the droplet, $\Delta E$, to be equal to the total energy across the boundary of the spherical droplet which can be expressed as:

$$
\Delta E=E_{\text {radiation }}+E_{\text {condensate }}-E_{\text {evaporate }}=\rho \frac{4}{3} \pi r^{3} c_{p} \frac{d T}{d t}
$$

where $\rho$ is the droplet density, $c_{p}$ is the specific heat of the droplet. The radiative energy from surrounding, $E_{\text {radiation }}$, energy condensed from surrounding gas molecules, $E_{\text {condensate, }}$ and the evaporated energy, $E_{\text {evaporate }}$, can be calculated by equations [9] to [11]:

$$
\dot{E}_{r a d}=\varepsilon s 4 \pi r^{2}\left(T_{s u r}^{4}-T^{4}\right)
$$

where $\varepsilon$ is the emissivity, $s$ is the Stefan-Boltzmann constant, $T_{s u r}$ is taken to be the average surrounding room temperature where the PP-MOCVD process is conducted, and $T$ is the droplet temperature.

$$
\dot{E}_{\text {condensate }}=\alpha \dot{N}_{\text {coll gas-drop }} e_{g}
$$

where $\alpha$ is the mass accommodation coefficient, $e_{g}$ is the molecular energy of the surrounding gas, $\dot{N}_{\text {coll gas-drop }}=\pi r^{2} \vec{V} n$ is the molecular collision rate between the droplet and the surrounding gas.

$$
\dot{E}_{\text {evaporate }}=\frac{d m}{d t} c_{p} T
$$

\section{Implementation of Droplet Model into QDS Algorithm}

The current droplet model is coupled to the QDS algorithm as a source condition to the gas phase flow simulation during the injection phase of the PP-MOCVD process.

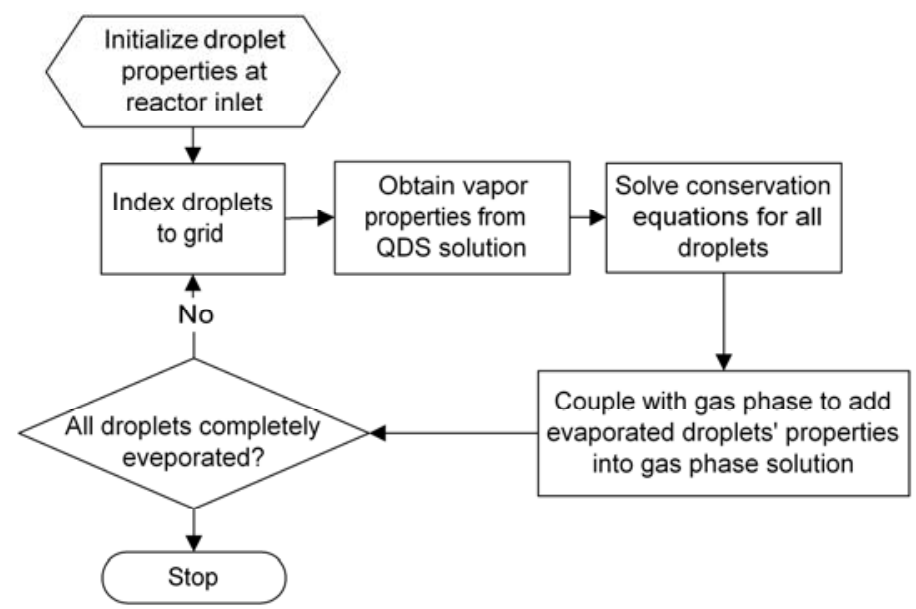

Figure 1. Flow chart of coupling the droplet model to gas phase solution by QDS solver 
Figure 1 shows the flow chart of the droplet model-QDS solver coupling. A representative number of droplets are initialized with droplet size and velocity selected stochastically from the log-normal distribution of those properties for a typical spray. Molecular properties of the evaporated droplets are calculated and added to the QDS gas phase solution while condensed gas phase molecules into the droplet due to gas-droplet collision add to the colliding droplet. A Lagrangian tracking method is used where the quantities of mass, momentum and energy exchanged with the vapour phase are multiplied by a factor during the droplet-gas coupling calculation in order to represent the actual quantity of droplets injected over the injection phase of the PP-MOCVD process.

\section{Results and discussion}

The liquid evaporation model coupled with QDS algorithm is tested by simulating the unsteady flow field in the PP-MOCVD process with the geometry shown in Figure 2.

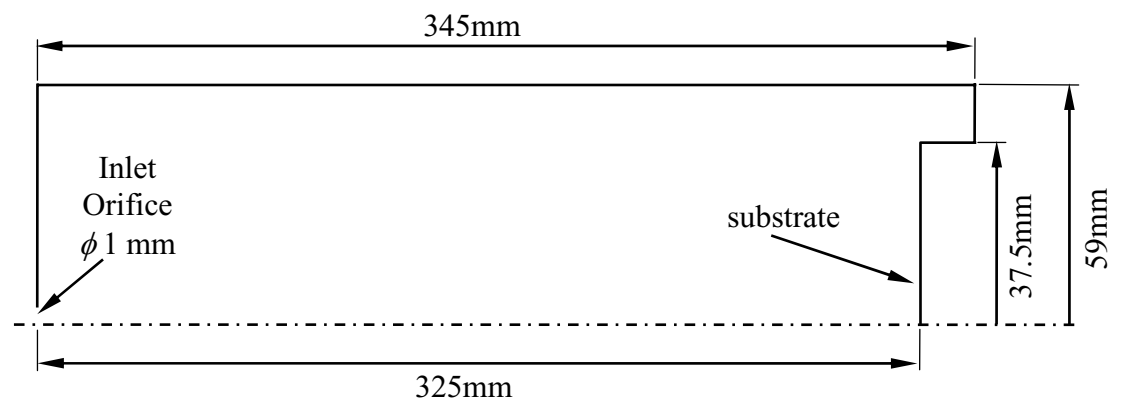

Figure 2. Schematic of Pulsed Pressure MOCVD reactor geometry.

Toluene (a common solvent in PP-MOCVD) with a ratio of specific heats of 1.089 and a gas constant of $R=90.2384 \mathrm{~J} /(\mathrm{kg} \cdot \mathrm{K})$ is chosen to be the simulation species for simplicity. As the precursor is very dilute in the injected solution, only the solvent is modelled. Since the intermolecular collision rate is high, it is assumed that the solvent and precursor are not spatially separated and the concentration of the precursor will be proportional to the concentration of the solvent at all locations and times. Twenty spherical simulated droplets at temperature of $323 \mathrm{~K}$ with diameter and velocities determined stochastically based on a log-normal distribution of typical spray (15) are injected into the reactor at each time step during the injection phase. The reactor is initially filled with stationary gas at $100 \mathrm{~Pa}$ and $293 \mathrm{~K}$. The droplet model is coupled to the second-order axisymmetric QDS solver (10). The simulations were carried out using 78,420 square cells with a total of 1000 simulated droplets. A multiplication factor of 208 is used to track the actual total droplet number injected into the reactor. This total number of droplets injected is determined from the mass of precursor solution used in the experimental investigation of the reactor. As a preliminary study and test of the developed droplet model, the simulation has been performed for a shorter time than the actual PP-MOCVD process time. In the current simulation, the injection phase was set to be $10 \mathrm{~ms}$ while pump down phase was $400 \mathrm{~ms}$. Experimental data given below was taken with an injection phase of one second and pump down phase of 10s. The simulation required approximately 16 hours on a $3.00 \mathrm{GHz}$ Intel Core 2 Duo CPU with 4GB of RAM.

Figure 3 shows the unsteady flow development of the gas phase solution in the reactor during one pulse. The evaporating droplets provide the only source of mass, 
momentum and energy to the flow field. These results show the increase in gas phase density which propagates through the reactor towards the substrate. In general, flash evaporation occurs when a sudden pressure drop results in a rapid evaporation of the liquid droplet. In the current simulation, as the simulated droplets at higher pressure enter the initially low pressure reactor, the droplets experience flash evaporation resulting in a rapid change in flow properties, such as density as shown in Figure 3. It is observed that, on average, all droplets are completely evaporated after being transported less than one third of the reactor length.

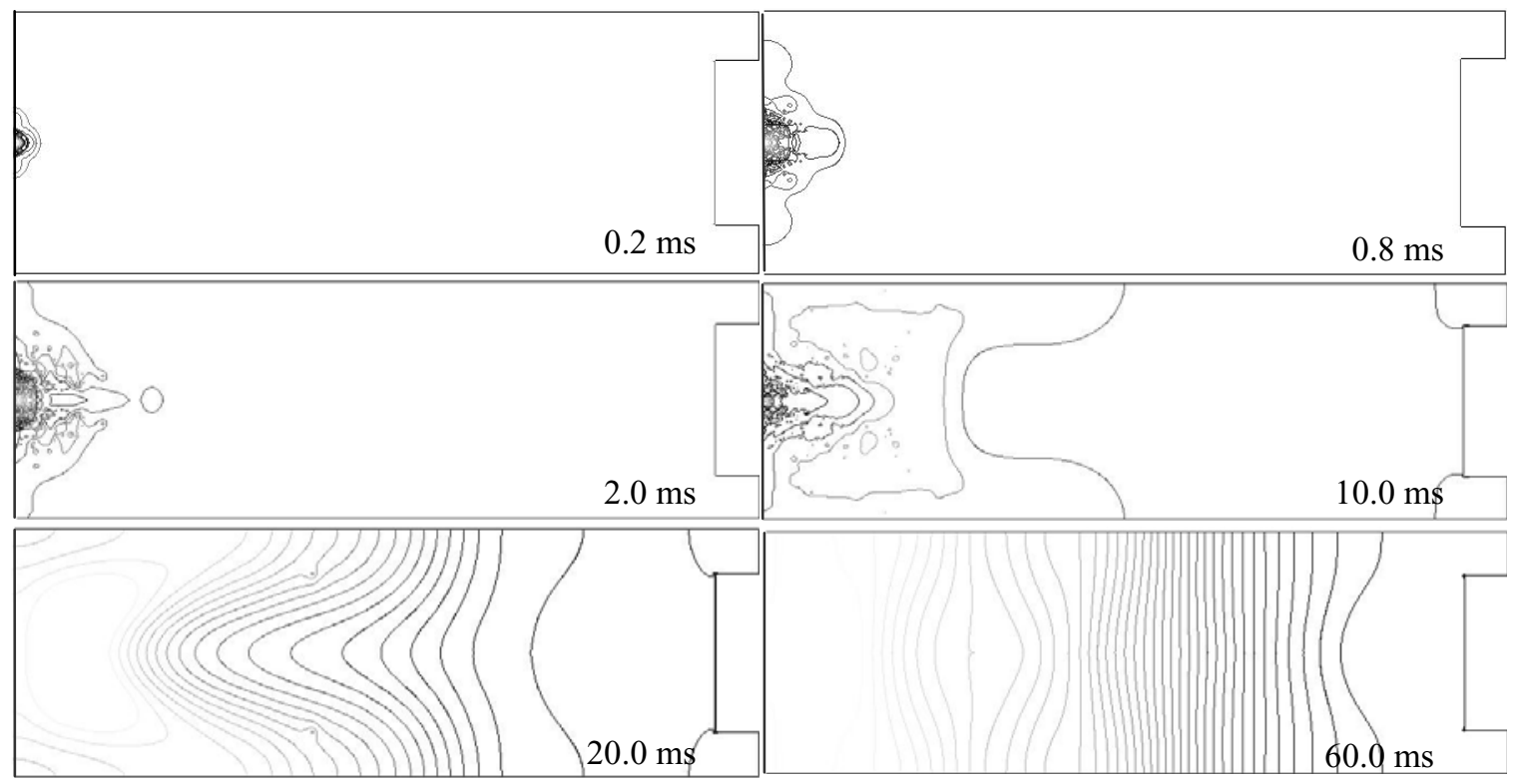

Figure 3. Contours of log (density) for the unsteady flow development in a PP-MOCVD reactor simulated with QDS coupled with droplet flash evaporation model.

Figure 4 shows the reactor pressure measured at a point near the substrate. The pressure rises rapidly during the injection phase. It should be noted that the actual process time of one pulse cycle was about 10s with one second of injection time. From the simulation result, although the pressure history during the pump down phase has a similar pattern to the experimental result as shown in Figure 4(a), the pressure profile is oscillatory. Three possible reasons have been identified: 1 . The droplets were injected into the reactor periodically, every $0.2 \mathrm{~ms}$ over the $10 \mathrm{~ms}$ injection period. This periodic injection of "fresh" droplets and the consequent evaporation results in sudden, periodic increases in vapour density. 2 . The flow rate at the reactor outlet is difficult to determine and has been assumed constant in the simulation. Thus, the low exhaust rate results in some of the particles being reflected back upstream after reaching the substrate and outlet wall, consequently oscillating lengthwise in the reactor. 3. The pressure oscillation in this simulation may be due to an acoustic resonance (organ pipe or Helmholtz). It is noted that the pressure oscillation during the pump down phase decays towards the end of the pump down phase. Therefore, the simulation result was able to show pressure rise during injection phase and pressure drop during pump down phase as indicated by the dashed line in Figure 4(b) compared to the experimental pressure profile.

The experimental data also show a shoulder at the beginning of the injection phase. The origin of this feature is not yet understood. It may be due to two distinct phases in 
the development of the spray, e.g. a period of coarse atomisation as the spray establishes, which is not modelled in the simulation.

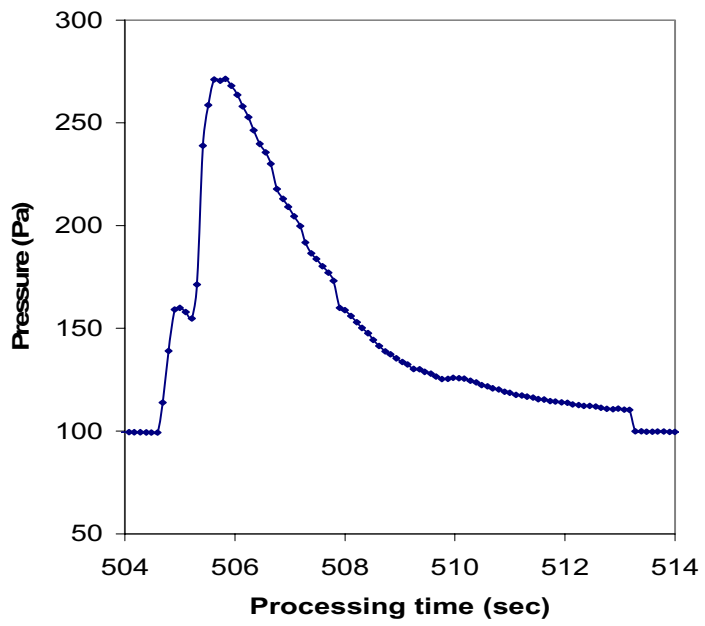

(a)

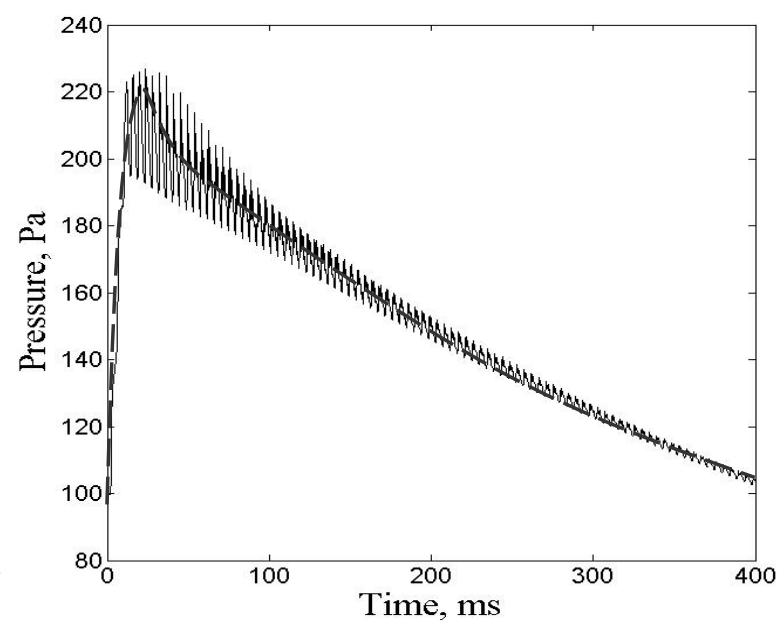

(b)

Figure 4. Comparison of experimental (a) and simulated (b) pressure change in near the substrate region during simulation time. Simulation and experiment used different injection pulse lengths and cycle periods.

Figure 5 shows the plots of mass flux of the precursor onto the substrate (MFLOS) and the accumulated mass of precursor onto the substrate (AMOS). As observed from experimental work, the deposition rate is highly dependent on the mass flux of the precursor transported near to the substrate which can be represented by MFLOS, while AMOS represents the total of mass that is being transported to the substrate. As the simulation domain represents an actual cylindrical PP-CVD reactor, the annular surface area of the substrate increases as substrate radius increases. Hence, to justify the uniformity of the total mass onto the substrate surface, AMOS over the substrate surface area was computed and plotted in Figure 5(b). Both plots suggest considerable uniformity in the deposited film.

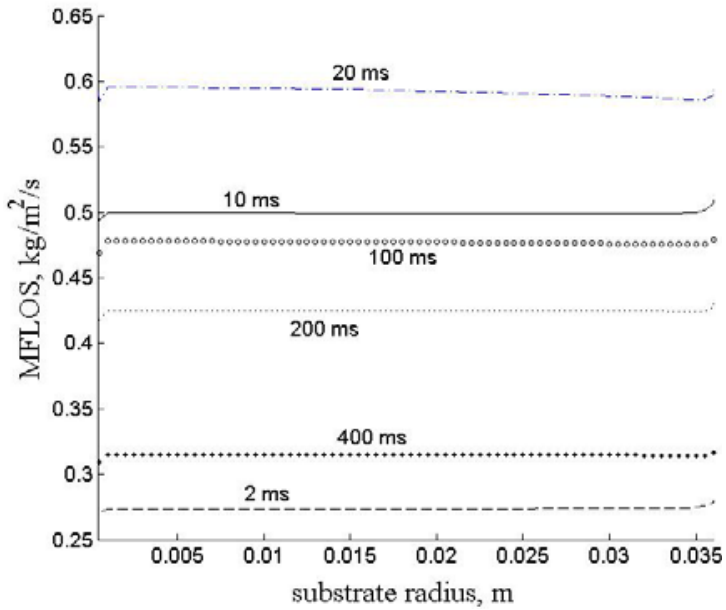

(a)

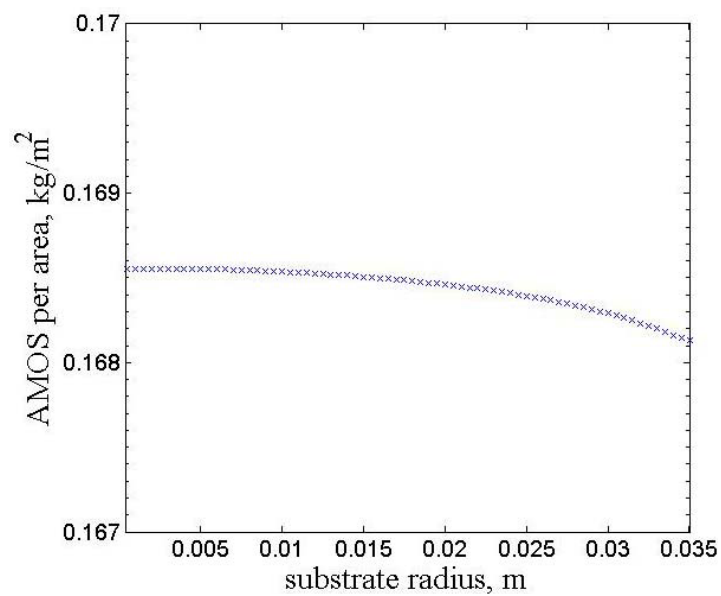

(b)

Figure 5. Mass flux of the precursor onto substrate (a) and accumulated mass of precursor onto substrate (b) in the radial direction of the substrate disc. 


\section{Conclusion}

In this paper a liquid droplet model was presented to provide a means to study the flash evaporation phenomena as well as the heat and mass transport of precursor droplets from the direct injection delivery system. This droplet model is coupled to the axisymmetric, second-order scheme QDS solver for flow simulation in a PP-MOCVD reactor. The preliminary work shows promising fast evaporation phenomena resulting in a rapid pressure rise in the reactor. The results also suggest good deposition uniformity. Simulations such as these will facilitate the design and optimization of PP-MOCVD reactors.

\section{Acknowledgments}

This research was conducted as part of Chin-Wai Lim's PhD project under the sponsorship of Universiti Tenaga Nasional, Malaysia. This work was also supported by the New Zealand Foundation for Research, Science and Technology (FRST) International Investment Opportunity Fund (IIOF) (grant no. UOCX070210).

\section{References}

1. H. Xie and R. Raj, Applied Physics Letters, 63(23), 3146 (1993).

2. V. A. Versteeg, C. T. Avedisian and R. Raj, Journal of the American Ceramic Society, 78(10), 2763 (1995).

3. S. P. Krumdieck, O. Sbaizero, A. Bullert and R. Raj, Surface and Coatings Technology, 167, 226 (2003).

4. S. P. Krumdieck and R. Raj, Journal of the American Ceramic Society, 82(6), 1605 (1999).

5. S. P. Krumdieck, A. Kristinsdottir, L. Ramirez, M. Lebedev and N. Long, Surface \& Coatings Technology, 201(22-23), 8908 (2007).

6. V. Siriwongrungson, M. M. Alkaisi and S. P. Krumdieck, Surface \& Coatings Technology, 201, 8944 (2007).

7. H. M. Cave, Development of Modelling Techniques for Pulsed Pressure Chemical Vapour Deposition (PP-CVD), Ph.D. Thesis, University of Canterbury (2008).

8. J. C. Maxwell, Collected Scientific Papers of James Clerk Maxwell, p. 625, Cambridge, UK (1890).

9. M. R. Smith, H. M. Cave, Y. -S. Chen, M. C. Jermy and J. -S. Wu, Journal of Computational Physics, 228, 2213 (2009).

10. H. M. Cave, M. R. Smith, J. -S. Wu, M. C. Jermy, S. P. Krumdieck, C. -W. Lim and K. -C. Tseng, Journal of Computational Physics, (in review 2009).

11. H. R. Pruppacher and J. D. Klett, Microphysics of Clouds and Precipitation, p. 506, Kluwer Academic Publishers, Boston (1997).

12. C. Dariva, L. A. F. Coelho and J. Vladimir Oliveira, Brazilian Journal of Chemical Engineering, 16(3), 1 (1999).

13. N. F. Carnahan and K. E. Starling, J. Chem. Phys., 51, 635 (1969).

14. R. Clift, J. R. Grace and M. E. Weber, Bubbles, Drops, and Particles, Academic Press, New York (1978).

15. E. Hervieu and T. Veneau, Journal of Loss Prevention in the Process Industries, 9(6), 413 (1996). 